Journal of Electronics and Informatics (2020)

Vol.02/ No. 02

Pages: 88-95

http://www.irojournals.com/iroei/

DOI: https://doi.org/10.36548/jei.2020.2.003

\title{
Trust-Value Based Wireless Sensor Network Using Compressed Sensing
}

\author{
JV Anand, \\ Assistant Professor, \\ ECE Department, \\ PACE Institute of Technology and Science, \\ Ongole, India. \\ Email ID: jvanand87@gmail.com
}

\begin{abstract}
Wireless sensor networks have quickly paved way to novel ways of communication between two nodes. They consist of sensor nodes that have the capacity to sense, communicate and compute. If a particular node in a WSN is not able to transmit data to the base station, routing algorithms will move into action to direct the data from the node. The proposed work deals with a routing algorithm based on trust awareness and compression sensing data, to handle data routing in a clustered WSN. In general, when sensor nodes have reduced overhead, compressed sensing is utilized for data aggregation. In order to strike a balance between number of messages transmitted, hop count, distance of transmission and the optimal trusted path, many nature inspired optimisation methods have been developed over the years. However, trust-based retrieval of compressed data is executed at the base station amidst malicious nodes.
\end{abstract}

Keywords: Wireless Sensor Networks; Trust Management; Compressed data; trust management; Optimal trusted path

\section{Introduction}

A Wireless sensor network is made up of many sensor nodes that can communicate with the base station as well as between themselves. The WSN routing protocol will vary based on the application and architecture. Due to the restriction in the sensor nodes' nature, topological changes and unexpected changes in node status, the routing protocol will be complicated. Depending on the architecture of the network be it location, hierarchical or flat-based routing, the protocol will be grouped. For locationbased routing, the location of the sensor node is used to determine the routing path. In hierarchical routing the notes are segregated into clusters. In flat routing, all the nodes are configured to have identical ability to route and to sense. Many protocols can be grouped as hybrid, reactive and proactive. In reactive protocol, all routes are computed based on demand and in proactive protocol, the routes are calculated even before they are called for. A combination of both these protocols are used in Hybrid protocol.

\section{Related works}

In [1] and [2] an evolutionary multi-path routing was developed with the idea to develop a trade-off between network life time and robustness. This work aimed to give the solution for energy hole problem in WSN. This algorithm proved to be extremely efficient in terms of energy and connectivity between 
Journal of Electronics and Informatics (2020)

Vol.02/ No. 02

Pages: 88-95

http://www.irojournals.com/iroei/

DOI: https://doi.org/10.36548/jei.2020.2.003

the various nodes was also preserved. In [3] a researcher proposed to provide an energy balanced routing based on position to maximize the network lifetime. This was accomplished by curtailed transmission that were not essential by forwarding search space. A routing algorithm based on multi-path rule driven protocol was proposed in [4] which was used in the biological immune system to drive the WSN. The protocol used records of routing paths and clusters to represent antibodies and antigens were represented by events. This resulted in increased network lifetime, data transmission and energy saving. Similarly in [5] a grid-based routing algorithm was proposed which had to ability to analyze the quality of communication of the node before hoping to the next one. In [6] using neighbourhood heuristics, a routing framework was developed. It was found that this methodology was able to integrate failover capability and route quality into one metric resulting in a more stable communication means. In [7] a review on the various network topologies were presented and a hierarchical routing protocol was analysed to determine its performance efficiency. This hierarchical routing comprised of area-based, tree-based, grid-based and chain-based routing.

In [8] the researcher has presented a routing algorithm based on particle swarm optimisation for software-defined WSN. Using this algorithm the distance of transmission was reduced further optimizing the amount of energy consumed. Authors in [8] have combined simulated annealing and genetic algorithm to increase the lifetime of the network for various applications. A comparison was drawn between this protocol and the distance-aware and energy-aware LEACH protocol and their performance was evaluated. In [9] another PSO-based algorithm which proved to be efficient in network lifetime, energy consumption and packet delivery. In [10] improved harmony search routing methodology was been introduced to develop a more efficient Wireless Sensor network. Similarly, distance aware routing and genetic algorithm have been used for optimised cluster head selection. The honeybees swarm intelligence-based routing proposed in [11] proves improved network lifetime and high power efficiency WSNs. In paper [12], a swarm intelligence based routing protocol using fuzzy logic is proposed to improve the lifetime of network.

\section{Proposed Methodology}

Based on the research on the work so far, there has been much emphasis on decreasing energy consume and increasing the life time. In the research paper, apart from satisfying these two basic conditions a routing algorithm that is based on Compressed sensing and trust is proposed in order to provide better security and reduce the message overhead is also proposed. Fig.1. depicts the methodology proposed. The objectives addressed in this work includes increasing the trust of path and minimizing the number of messages, number of hops and the travel distance. Some of the already existing algorithms that are inspired by nature are firefly algorithm, differential evolution (DE), ant colony optimization (ACO), artificial bee colony (ABO) and particle swarm optimization (PSO) routing which are also used to validate the proposed routing methodology using trust aware routing. Data aggregation is performed at $\mathrm{CH}$ and the reconstruction is performed based on trust. 
Journal of Electronics and Informatics (2020)

Vol.02/ No. 02

Pages: 88-95

http://www.irojournals.com/iroei/

DOI: https://doi.org/10.36548/jei.2020.2.003

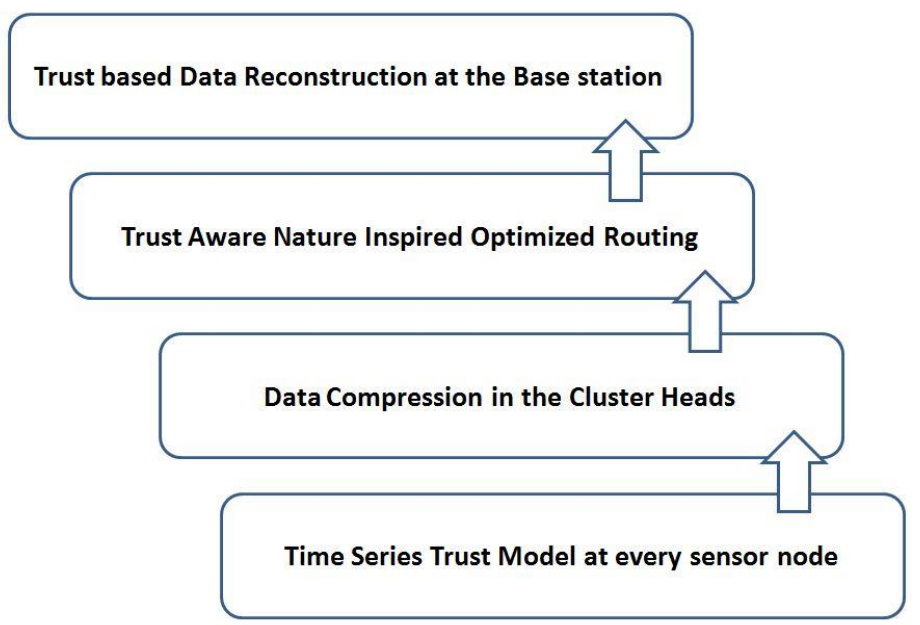

Fig.1. Proposed Trust-based Methodology

\subsection{Trust Series}

In WSN trust is a measure of reliability of the node based on capability, node availability, path connectivity and data authenticity. The application and management of trust models have been reviewed in [13]. Some application of WSN include node selection, localization, data aggregation, secure routing and malicious attack detection. Based on fuzzy logic, a fully distributed trust management system has been developed in [14] which had the ability to identify abnormal node behaviour to increase forecast trust, network performance and also to improve cooperation. Based on the resource utilized as well the energy consumed, the reputation of trust of any node is evaluated. beta distribution can be used to describe the node's credibility as shown in [15]. An investigation was held on linguistic fuzzy as well as bio-inspired reputation and trust model and it was concluded that path length factors and pheromone played an important role in these models, resulting in an improved heterogeneous WSN. When the WSN is observing time series data such as smart grid, environmental data etc. the trust value of a node can be defined at a specific instance. The distritbution of the values are considered to determine the node trust value. Relative trust (REtrust), ENtrust, data trust (DAtrust) energy trust (ENtrust) are the terms used to define the trust of a node.

$$
\begin{gathered}
E(d)=d^{\delta}+C \\
\text { DAtrust }=\int_{v_{d}}^{\infty} f(x) d x
\end{gathered}
$$

where $f(x)=\frac{1}{\sigma \sqrt{2} \pi} e^{\frac{-(x-\mu)^{2}}{2 \sigma^{2}}}, \mathrm{C}$ represents a constant for radio signal, $\delta$ is the attenuation factor, $\mathrm{d}$ is the distance and $\mathrm{x}$ is the attribute value. 
Journal of Electronics and Informatics (2020)

Vol.02/ No. 02

Pages: 88-95

http://www.irojournals.com/iroei/

DOI: https://doi.org/10.36548/jei.2020.2.003

With the help of limited random linear measurement, CS techniques are used to build information and reconstruct sparse signals in an accurate manner that is found using the sampling rate of the sparse signals. When WSN includes CS methodology, it will lead to notable decrease in energy management, traffic, time delay and power consumption.

\subsection{Data Aggregation and Reconstruction using Trust}

Data is detected from the nodes and this data is further sent to $\mathrm{CH} . \emptyset_{i}$ is the measurement matrix generated which is in $M_{i} \times N_{i}$ where $z_{i}=\emptyset_{i} y_{i}$. Within a specific cluster, the measurement matrix of the nodes are known to the $\mathrm{CH}$ nodes. This type of data aggregation based on clusters will help to formulate Block Diagonal Matrices where a relationship is established between the iid Gausian entries, $\mathrm{i}^{\text {th }}$ cluster and $\mathrm{i}^{\text {th }}$ block. This matrix has the ability to satisfy the condition required for recovering data accurately. Using the CS reconstruction algorithm, the sensor data are recovered by sending $\mathrm{M}$ measurements to the base station from the $\mathrm{CH}$. From the measurements, it is possible to reconstruct ksparse signal with the help of 11- optimisation problem as shown in the equation below.

$$
\hat{\theta}=\arg || \theta|| \text { where } \mathrm{y}=\varnothing \varphi \theta
$$

From the compressed data obtained, the original data is extracted by the base station. In the proposed work, the trust model formulated by us is used to reconstruct the data based on the trust value. Transmission between the clusters make use of CS and routing between the clusters is performed using a novel multi-hop system. The proposed technique is based on ABC and PSO in addition to CS in order to form the WSN routes.

\subsection{Clustering in WSN}

Reduction of redundant and uncorrelated data, conservation of communication bandwidth and increasing network scalability are some of the advantages of clustering in WSN. The proposed algorithm has its roots in k-means clustering algorithm which uses minimum distance criteria to build clusters of identical size. The errors present inside the clusters are eliminated using this method. The objective function can be expressed as the equation given below:

$$
J=\sum_{j=1}^{k} \sum_{i=1}^{n}\left\|y_{i}^{(j)}-c_{j}\right\|^{2}
$$

where $c_{j}$ represents the cluster centers, $y_{i}^{(j)}$ is the place of data measurement and $\left\|y_{i}^{(j)}-c_{j}\right\|^{2}$ is the distance measured between the data and the point. 
Journal of Electronics and Informatics (2020)

Vol.02/ No. 02

Pages: 88-95

http://www.irojournals.com/iroei/

DOI: https://doi.org/10.36548/jei.2020.2.003

\section{Results and Discussion}

At the base station, the compressed data is reconstructed based on trust value during data aggregation. This is further done at the presence of 10-25\% malicious nodes such that the Normalised mean squared error (NSME) can be determined at the base station. NSME can be calculated such that

$$
N S M E=\frac{\frac{1}{N} \sum_{i=1}^{N}\left(H_{e}(i)-H_{a}(i)\right)^{2}}{\left(\frac { 1 } { N } \sum _ { i = 1 } ^ { N } ( H _ { e } ( i ) ) \left(\frac{1}{N} \sum_{i=1}^{N}\left(H_{a}(i)\right)\right.\right.}
$$

Where $\mathrm{H}_{\mathrm{a}}$ is the observed varioable, $\mathrm{H}_{\mathrm{e}}$ is the estimated variable and $\mathrm{N}$ is the input data. For a specific cluster, the reconstruction of temperature data is represented in Fig.2. The observed readings indicated that the least NMSE recording is made when using the proposed trust-based reconstruction.

\section{Temperature Data}

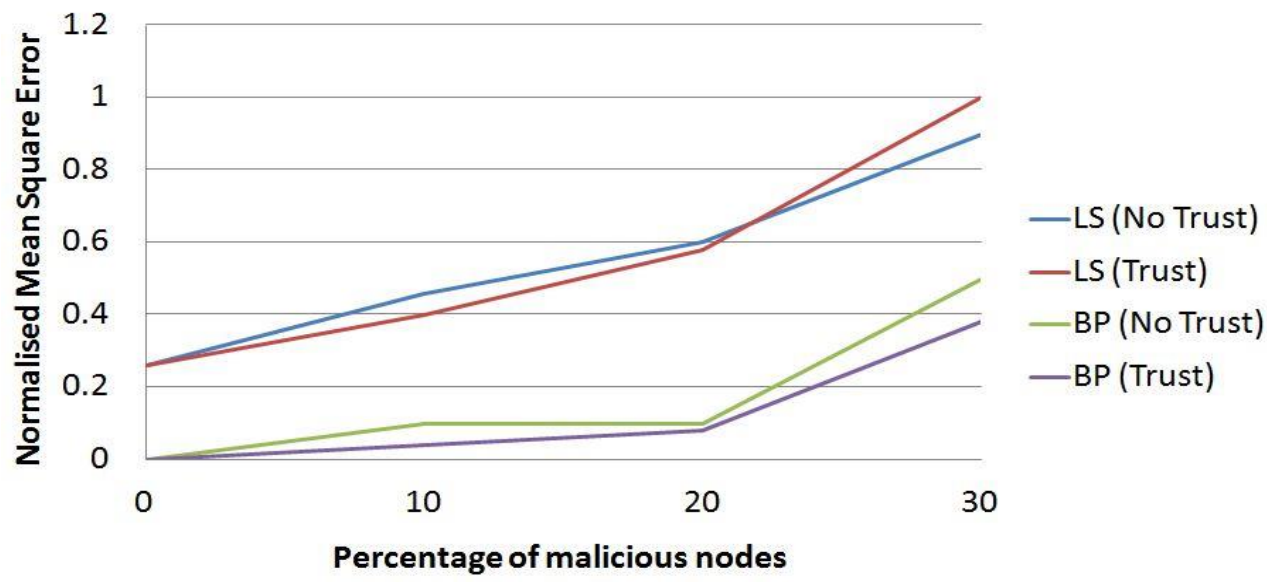

Fig.2. Trust Based Reconstruction of Data

The average trust value for various nodes in WSN such that $\mathrm{CS}$ is performed for $\mathrm{CR}=3$ is represented in Fig. 3 and the energy consumed for $\mathrm{CR}=3$ is represented in Fig. 4 . 
Journal of Electronics and Informatics (2020)

Vol.02/ No. 02

Pages: 88-95

http://www.irojournals.com/iroei/

DOI: https://doi.org/10.36548/jei.2020.2.003

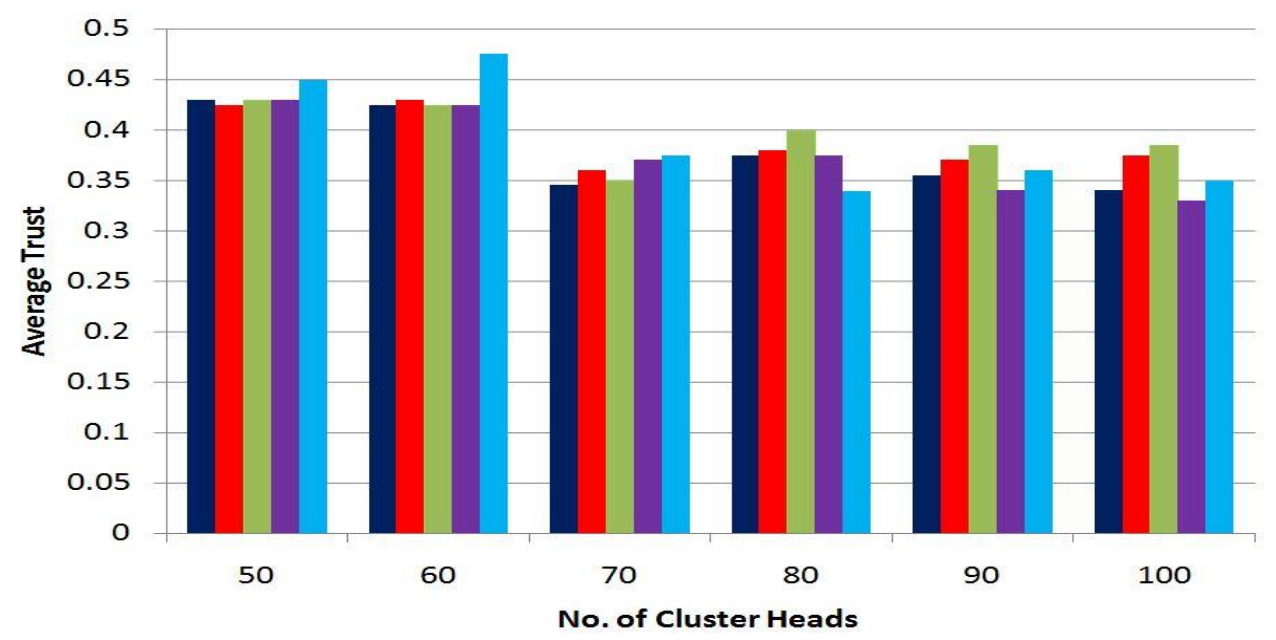

Fig.3. Average Trust for $\mathrm{CS}$ when $\mathrm{CR}=3$

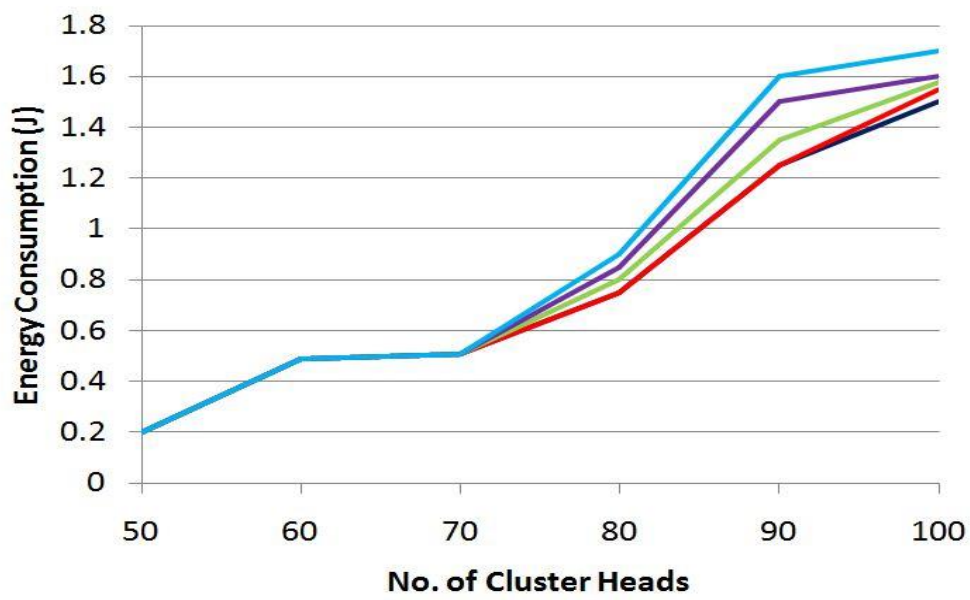

Fig.4. Energy consumed with CS when $\mathrm{CR}=3$

\section{Conclusions}

The proposed research work is based clustered WSN that can be routed using CS and trust awareness. There are 5 optimisation methods based on which the methodology is evolved and is focused on maximisation of trust of the route, minimization of message overhead, number of hops and maximum distance. When CS is implemented, it is observed that the performance of WSN is improved in terms of energy efficiency and losses deliver. Maximizing the trust of the routing and decreasing the total hops taken is performed best by the proposed methodology. Our paper indicates that using the maximum trusted path, 10-25\% malicious nodes can be determined. 
Journal of Electronics and Informatics (2020)

Vol.02/ No. 02

Pages: 88-95

http://www.irojournals.com/iroei/

DOI: https://doi.org/10.36548/jei.2020.2.003

\section{References}

[1] Ari, A.A.A., Yenke, B.O., Labraoui, N., Damakoa, I. and Gueroui, A. (2016) 'A power efficient cluster-based routing algorithm for wireless sensor networks: honeybees swarm intelligence based approach' , Journal of Network and Computer Applications, Vol. 69, pp.77-97.

[2] Xiang, W., Wang, N. and Zhou, Y. (2016) 'An energy-efficient routing algorithm for software-defined wireless sensor networks' , IEEE Sensors Journal, Vol. 16, No. 20, pp.7393-7400.

[3] Edwin Prem Kumar, G., Baskaran, K., Elijah Blessing, R. and Lydia, M. (2018) 'Trust based data prediction, aggregation and reconstruction using compressed sensing for clustered wireless sensor networks', Computer and Electrical Engg.

[4] Singh, S., Verma, V.K. and Pathak, N.P. (2015) 'Sensors augmentation influence over trust and reputation models realization for dense wireless sensor networks' , IEEE Sensors Journal, Vol. 15, No. 11, pp.6248-6254.

[5] Bhatia, T., Kansal, S., Goel, S., Verma, A.K. (2016) 'A genetic algorithm based distanceaware routing protocol for wireless sensor networks', Computers and Electrical Engineering, Vol. 56, pp.441-455.

[6] Delaney, D.T., Higgs, R., O’ Hare, G.M.P. (2014) 'A stable routing framework for tree based routing structures in WSNs' , IEEE Sensors Journal, Vol. 14, No. 10, pp.35333547.

[7] Edwin Prem Kumar, G., Baskaran, K., Elijah Blessing, R. and Lydia, M. (2016) Evaluation of hybrid trust models using ant colony optimization in wireless sensor networks' , Intl. J. Smart Sensing and Intelligent Systems, Vol. 9, No. 3, pp.1243-1260.

[8] Fang, W., Zhang, C., Shi, Z., Zhao, Q. and Shan, L. (2016) 'BTRES: beta-based trust and reputation evaluation system for wireless sensor networks' , Journal of Network and Computer Applications, Vol. 59, pp.88-94.

[9] Nguyen, M.T. and Teague, K.A. (2017) 'Compressive sensing based random walk routing in wireless sensor networks' , Ad Hoc Networks, Vol. 54, pp.99-110.

[10] Rahat, A.A.M., Everson, R.M. and Fieldsend, J.E. (2016) 'Evolutionary multi-path routing for network lifetime and robustness in wireless sensor networks', Ad Hoc Networks, Vol. 52, pp.130-145.

[11] Rathore, H., Badarla, V. and George, K.J. (2016) 'Sociopsychological trust model for wireless sensor networks' , Journal of Network and Computer Applications, Vol. 62, pp.75-87.

[12] Raj, J. S. (2020). Machine Learning Based Resourceful Clustering With Load Optimization for Wireless Sensor Networks. Journal of Ubiquitous Computing and Communication Technologies (UCCT), 2(01), 29-38.

[13] Raj, J. S. ENERGY EFFICIENT SENSED DATA CONVEYANCE FOR SENSOR NETWORK UTILIZING HYBRID ALGORITHMS. 
Journal of Electronics and Informatics (2020)

Vol.02/ No. 02

Pages: 88-95

http://www.irojournals.com/iroei/

DOI: https://doi.org/10.36548/jei.2020.2.003

[14] Haoxiang, W., \& Smys, S. (2020). Soft Computing Strategies for Optimized Route Selection in Wireless Sensor Network. Journal of Soft Computing Paradigm (JSCP), 2(01), $1-12$.

[15] Raj, J. S. (2019). QoS optimization of energy efficient routing in IoT wireless sensor networks. Journal of ISMAC, 1(01), 12-23.

\section{Authors Biography}

JV Anand, is an Assistant Professor, ECE Department, at PACE Institute of Technology and Science, Ongole, India his major area of research are semiconductor materials, computer science, nano electronics, object/web technologies, microelectronics, quantum electronics, VLSI, electronic system design, IT integrated manufacturing, fabrication, and analysis 